\title{
EDITORIAL
}

\section{Myocardial dysfunction in sepsis: no role for $\mathrm{NO}$ ?}

\author{
E Belcher, J Mitchell, T Evans
}

Heart 2002;87:507-509

The effects of nitric oxide on myocardial function in clinical sepsis are unclear, with studies in experimental models suggesting both beneficial and deleterious effects

S epsis and its sequelae ${ }^{1}$ represent an important cause of mortality among the critically ill, particularly if cardiovascular dysfunction ensues. In these circumstances, refractory hypotension develops leading to multiple organ failure, and some $50 \%$ of such cases fail to survive. $^{2}$ Although sepsis is defined as the systemic response to infection, less than $50 \%$ of patients with septic shock have positive blood cultures. ${ }^{3}$ Indeed, the clinical manifestations of sepsis may be seen in association with a variety of non-infective insults including major trauma, burns, pancreatitis, rhabdomyolysis, and surgery necessitating cardiopulmonary bypass, and are then termed the systemic inflammatory response syndrome (SIRS).

\section{MYOCARDIAL DYSFUNCTION IN SEPSIS}

Traditionally, descriptions of septic shock defined an early, hyperdynamic phase, with warm peripheries, low systemic vascular resistance (SVR), and high cardiac output. In non-survivors the "warm" phase was thought to progress to a hypodynamic or "cold" phase in which cool peripheries, increased SVR, and low cardiac output were the dominant clinical features. However, traditional indices of myocardial function, including cardiac and stroke volume index and ejection fraction, may be inaccurate as a significant depression in contractility can be masked by changes in heart rate, preload, and afterload. To quantify intrinsic myocardial depression, an assessment of left ventricular performance using load independent techniques is therefore required. The end systolic pressure-volume relationship (ESPVR) is unaffected by changes in afterload and preload over a wide range. The slope is maximal at end systole $\left(\mathrm{E}_{\max }\right)$ and has the units of elastance $\mathrm{E}=\Delta \mathrm{P} / \Delta \mathrm{V}$. The linear relationship between these parameters is shifted to the left under conditions of increased contractility and to the right with reduced contractility.

Such techniques have revealed that patients with septic shock have severely depressed ejection fraction with ventricular dilatation, as measured by an increase in mean systolic and end diastolic ventricular volumes. In survivors, these changes reverse over a period of some 7-10 days, ${ }^{4}$ suggesting that they form part of a compensatory mechanism in which acute dilatation of the left ventricle, without changes in pressure, leads to an increase in left ventricular compliance. Conceivably, this may enable patients to maintain a normal stroke volume and cardiac indices, despite a profound loss of myocardial contractility, via the Frank-Starling mechanism (fig 1). ${ }^{5}$ By contrast, non-survivors develop diastolic dysfunction with a reduced left ventricular end diastolic volume (LVEDV) despite evidence of increased left atrial pressure. This stiffness impairs diastolic filling and therefore stroke volume. Similar changes in right ventricular performance have been detected. ${ }^{6}$

\section{ROLE OF NITRIC OXIDE}

The pathogenesis of depressed myocardial dysfunction in sepsis is incompletely understood, but recent clinical studies have provided insight into the potential role of the endogenous vasodilator nitric oxide (NO) in this process. NO is synthesised from the semi-essential amino acid L-arginine by three isoforms of NO synthase (NOS). Under physiological conditions, NO is produced by constitutive (cNOS) enzymes expressed predominantly in endothelial cells (eNOS) and neurons (nNOS) respectively. However, other cell types, including myocytes and endocardial cells, have also been shown to express NOS constitutively. ${ }^{78}$ Under conditions of stress, including sepsis, induction of inducible NOS (iNOS) leads to the production of large amounts of NO. Since NO is the principal mediator of vascular smooth muscle tone, its role in causing profound systemic vasodilatation in septic shock seems logical.

By contrast, the role of NO in the pathogenesis of myocardial dysfunction in sepsis is less clear cut, although potential mechanisms exist. NO stimulates the enzyme soluble guanylate cyclase that converts guanosine triphosphate (GTP) to $3^{\prime}, 5^{\prime}$ cyclic guanosine monophosphate (cGMP). In the cardiac myocyte cGMP inhibits the $\beta$ adrenergic stimulated increase in the slow inward calcium current and reduces the calcium affinity of the contractile apparatus. ${ }^{9} \mathrm{NO}$ also reduces cAMP via phosphodiesterase $\mathrm{e}^{10}$ and alters protein kinase activity and L type calcium channels. ${ }^{11}$ Besides these direct adverse effects on myocardial contractility, NO inhibits mitochondrial respiration and therefore adenosine triphosphate production within the myocardium. Cellular damage may also occur following binding with reactive oxygen species to form the toxic radical peroxynitrite. At high concentrations NO also leads to apoptosis of cardiomyocytes. ${ }^{12}$ Moreover, molecular studies using immunohistochemistry, reverse transcription polymerase chain reaction, and in situ

Abbreviations: EDPVR, end diastolic pressure-volume relationship; ESPVR, end systolic pressure-volume relationship; LVEDV, left ventricular end diastolic volume; MLA, monophosphoryl lipid A; NO, nitric oxide; NOS, nitric oxide synthase; SIRS, systemic inflammatory response syndrome; SVR, systemic vascular resistance 


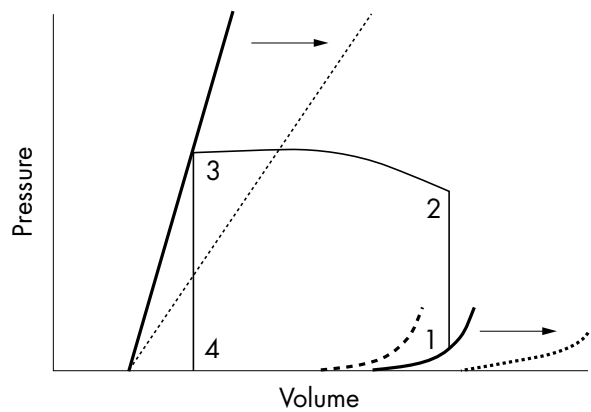

Figure 1 Left ventricular pressure-volume relationships. ${ }^{5}$ The bold straight line shows the normal end systolic pressure-volume relationship (ESPVR), defining ventricular contraction. ESPVR is independent of changes in preload and afterload. The slope is maximal at end systole and has the units of elastance, $E=\Delta P / \Delta V$. It has therefore been designated $E_{m a x}$. The bold curved line shows normal end diastolic pressure-volume relationship (EDPVR). The straight section of the line represents addition of the initial volume. While ventricular volume increases from zero to approximately $50 \mathrm{ml}$, transmural pressure remains at zero. Left ventricular end diastolic pressure (LVEDP) then increases in a curvilinear manner during addition of further volume. The pressure-volume loop (1-4) represents one cardiac cycle (1-2, isovolumetric contraction; 2-3, stroke volume; $3-4$, isovolumetric relaxation). The interrupted straight line represents $E_{\max }$ in sepsis, with depressed contractility indicated by a downward and right shift of the ESPVR. In survivors of septic shock this is accompanied by a right shift of the EDPVR (interrupted curved line) caused by ventricular dilatation, enabling an increase in stroke volume. In non-survivors diastolic stiffness develops represented by an upward and left shift of the EDPVR (bold interrupted curved line), leading to impaired diastolic filling and a reduction in stroke volume. Arrows represent possible contribution of nitric oxide (NO) to the pressure-volume relationships in sepsis.

While NO may be responsible for the reduction in systolic contractility, the septic patient is able to maintain end systolic volume because of a reduction in systemic vascular resistance; therefore the overriding haemodynamic effect of $\mathrm{NO}$ in sepsis may be facilitation of ventricular dilatation, which acts to further stroke volume and maintain cardiac output. Potentially, NO blockade in sepsis may convert the EDPVR of a surviving septic patient to a non-survivor.

hybridisation have confirmed a sepsis induced release of NO in the microvasculature and cardiomyocytes in endotoxaemic rats. ${ }^{13}$ Early animal studies also supported the concept of a causal relation between NO release and myocardial depression in sepsis. NO decreased contractility in isolated cardiac myocytes, ${ }^{14}$ in isolated papillary muscle preparation, ${ }^{15}$ and in the isolated working heart. ${ }^{16}$

The existence of potential mechanisms for cardiac depression and the results of functional studies therefore make therapeutic NOS inhibition in sepsis theoretically attractive. Encouraging early clinical trials demonstrated that inhibition of both the constitutive and inducible isoforms of NOS in patients with sepsis increased SVR with a concomitant reduction in the need for pressor agents. ${ }^{17}$ Unfortunately, a subsequent investigation powered to show a mortality benefit in a similar patient population was stopped prematurely after NOS inhibition using the same agent administered for longer periods was associated with a significant increase in mortality, attributable to an excess of cardiovascular deaths. ${ }^{18}$

Why this clinical trial demonstrated an apparently detrimental effect on cardiovascular function in these circumstances was not clear. However, it appears that the effect of NO on cardiac function in sepsis is more complex than the purely deleterious role first hypothesised. Evidence is emerging that NO derived from cNOS has beneficial effects in the heart. Physiological concentrations of NO do not have acute negative inotropic effects $^{19}$ and (up to $1 \mu \mathrm{M}$ ) are known to support myocardial performance. ${ }^{20}$ Furthermore, in human septic shock myocardial perfusion is normal or increased ${ }^{21}$ possibly because eNOS related biosynthesis of $\mathrm{NO}$ in the coronary vasculature exerts a beneficial effect by counteracting vasoconstriction induced by other vasomotor substances, such as the endothelins, which are also produced under inflammatory conditions. ${ }^{22}$ Indeed, in this and other animal models of sepsis, inhibition of constitutive derived NO has been associated with significant myocardial depression, although in this and other investigations the experimental techniques employed have not evaluated $\mathrm{NO}$ release, only protein transcription and expression.

If constitutive derived NO is beneficial, is selective iNOS inhibition the answer? Probably not, in that the concept of a detrimental effect of iNOS derived NO on myocardial function in sepsis is also questionable. Not only is direct evidence for a link between iNOS expression and diminished contractility lacking, but inducible NO release may even have beneficial effects on myocardial performance. Should the ventricular dilatation seen in survivors of septic shock be NO mediated, a further mechanism for the detrimental effects of NO inhibition in sepsis would exist. Data from our own laboratory suggest that the myocardial hyporeactivity seen in endotoxin induced sepsis is reversed by the administration of L-arginine. In this model, NO is therefore cardioprotective. ${ }^{23}$ Similarly, in a porcine model, inhalation of NO prevented endotoxin induced left ventricular impairment. ${ }^{24}$ The idea that iNOS induction in sepsis may not be solely detrimental to myocardial function is paralleled by studies of late phase ischaemic preconditioning, which show that NO may play a pivotal role in myocardial protection as both a trigger and mediator of this phenomenon..$^{25}$ Interestingly, monophosphoryl lipid A (MLA), a detoxified derivative of lipopolysaccharide

Table 1 Proposed beneficial and adverse effects of nitric oxide (NO) on myocardial function in sepsis

Potential cardiodepressant effects of $\mathrm{NO}$

(1) Alterations in protein kinase activity and then L type calcium channel

(2) Decrease in the myofibril response to calcium

(3) Decreased cAMP via phosphodiesterase

(4) Direct inhibition of mitochondrial respiration and therefore adenosine triphosphate production within the myocardium

(5) Stimulation of cellular damage following binding with reactive oxygen species to form peroxynitrite

(6) Triggering of apoptosis in cardiomyocytes

Potential cardioprotective effects of NO

(1) Increased myocardial perfusion due to vasodilatory effect

(2) Free radical scavenger reducing ischaemia reperfusion injury via: -inhibition of platelet aggregation -inhibition of leucocyte adhesion to endothelial cells -stabilisation of cell membranes

(3) Mediation of ventricular dilatation allowing utilisation of Frank-Starling mechanism

(4) Antiarrhythmic properties of NO

(5) Macrophage activation leading to bacterial lysis 
from Gram negative bacteria, is able to produce delayed protection against myocardial infarction. NO has been implicated in the delayed preconditioning induced by ischaemia and by MLA. ${ }^{26}$ In this setting, iNOS induced NO is therefore not only cardioprotective, but may represent a protective mechanism for the protection evolved by an agent derived from lipopolysaccharide, a major mediator of septic shock.

\section{NO WAY FORWARD FOR NO?}

The effects of NO on myocardial function in clinical sepsis remain unclear. Studies in experimental models suggest both beneficial and deleterious effects (table 1). While inhibition of NOS may improve systolic cardiac function and vasomotor tone, the beneficial effects of $\mathrm{NO}$ on diastolic function and myocardial perfusion would be lost. This knowledge alone is sufficient to justify further work to elucidate the possible therapeutic benefits of manipulation of NO production in sepsis.

\section{ACKNOWLEDGEMENTS}

EB is supported by the Medical Research Council. JM is supported by the Wellcome Trust

\section{Authors' affiliations}

E Belcher, J Mitchell, T Evans, Unit of Critical Care, Imperial College School of Medicine, Royal Brompton Hospital, London SW3 6NP, UK

\section{REFERENCES}

1 American College of Chest Physicians/Society of Critical Care Medicine. Consensus conference: definitions for sepsis and organ failure and guidelines for the use of innovative therapies in sepsis. Crit Care Med 1992; 20:864-74

2 Friedman G, Silva E, Vincent JL. Has the mortality of septic shock changed with time. Crit Care Med 1998;26:2078-86.

3 Danner RL, Elin RJ, Hosseini JM, et al. Endotoxemia in human septic shock. Chest 1991:99:169-75.

4 Parker MM, Shelhamer JH, Bacharach SL, et al. Profound but reversible myocardial depression in patients with septic shock. Ann Intern Med 1984;100:483-90.

5 Walley KRW. In: Hall JBS, Wood GA, ed. Principles of critical care. New York: McGraw-Hill, Inc, 1992:1393-416.

6 Kimchi A, Ellrodt AG, Berman DS, et al. Right ventricular performance in septic shock: a combined radionuclide and hemodynamic study. J Am Coll Cardiol 1984;4:945-51.

7 Balligand JL, Ungureanu-Longrois D, Simmons WW, et al. Induction of $\mathrm{NO}$ synthase in rat cardiac microvascular endothelial cells by IL-1 beta and IFN-gamma. Am J Physiol 1995;268(3 Pt 2):H1293-303.
8 Wildhirt SM, Dudek RR, Suzuki H, et al. Immunohistochemistry in the identification of nitric oxide synthase isoenzymes in myocardial infarction. Cardiovasc Res 1995;29:526-31.

9 Shah AM, Spurgeon HA, Sollott SJ, et al. 8-bromo-cGMP reduces the myofilament response to $\mathrm{Ca} 2+$ in intact cardiac myocytes. Circ Res 1994:74:970-8.

10 Fischmeister R, Hartzell HC. Cyclic AMP phosphodiesterases and Ca2+ current regulation in cardiac cells. Life Sci 1991;48:2365-76.

11 Wahler GM, Dollinger SJ. Nitric oxide donor SIN-1 inhibits mammalian cardiac calcium current through cGMP-dependent protein kinase. Am J Physiol 1995;268(1 Pt 1):C45-54.

12 Kawaguchi $\mathbf{H}$, Shin WS, Wang Y, et al. In vivo gene transfection of human endothelial cell nitric oxide synthase in cardiomyocytes causes apoptosis-like cell death. Identification using Sendai virus-coated liposomes. Circulation 1997;95:2441-7.

13 Ishiwata T, Guo F, Naito Z, et al. Differential distribution of ecNOS and iNOS mRNA in rat heart after endotoxin administration. Jpn Heart J 1997:38:445-55.

14 Brady AJ, Warren JB, Poole-Wilson PA, et al. Nitric oxide attenuates cardiac myocyte contraction. Am J Physiol 1993;265(1 Pt 2):H176-82.

15 Mohan P, Brutsaert DL, Paulus WJ, et al. Myocardial contractile response to nitric oxide and cGMP. Circulation 1996:93:1223-9.

16 Sun X, Wei S, Szabo C, et al. Depression of the inotropic action of isoprenaline by nitric oxide synthase induction in rat isolated hearts. Eur Pharmacol 1997;320:29-35

17 Grover R, Zaccardelli D, Colice G, et al. An open-label dose escalation study of the nitric oxide synthase inhibitor, N(G)-methyl-L-arginine hydrochloride (546C88), in patients with septic shock. Glaxo Wellcome international septic shock study group. Crit Care Med 1999;27:913-22.

18 Grover R, Lopez A, Lorente J, et al. Multi-center, randomised, placebo-controlled, double blind study of the nitric oxide synthase inhibitor 546C88: effect on survival in patients with septic shock. Crit Care Med 1999:27(1 suppl):33A.

19 Weyrich AS, Ma XL, Buerke M, et al. Physiological concentrations of nitric oxide do not elicit an acute negative inotropic effect in unstimulated cardiac muscle. Circ Res 1994;75:692-700.

20 Muller-Strahl G, Kottenberg K, Zimmer HG, et al. Inhibition of nitric oxide synthase augments the positive inotropic effect of nitric oxide donors in the rat heart. J Physiol 2000;522(Pt 2):311-20.

21 Cunnion RE, Schaer GL, Parker MM, et al. The coronary circulation in human septic shock. Circulation 1986;73:637-44.

22 Grandel U, Sibelius U, Schrickel J, et al. Biosynthesis of constitutive nitric oxide synthase-derived nitric oxide attenuates coronary vasoconstriction and myocardial depression in a model of septic heart failure induced by Staphylococcus aureus alpha-toxin. Crit Care Med 2001;29:1-7.

23 Price S, Evans T, Mitchell JA. Atrial dysfunction induced by endotoxin in rats is modulated by L-arginine: role of nitric oxide. $\mathrm{Br} J$ Pharmacol 1999;(77P)

24 Ishihara S, Ward JA, Tasaki O, et al. Inhaled nitric oxide prevents left ventricular impairment during endotoxemia. J Appl Physiol 1998;85:2018-24

25 Rakhit RD, Edwards RJ, Marber MS. Nitric oxide, nitrates and ischaemic preconditioning. Cardiovasc Res 1999;43:621-7.

26 Zhao L, Elliott GT. Pharmacologic enhancement of tolerance to ischemic cardiac stress using monophosphoryl lipid A. A comparison with antecedent ischemia. Ann N Y Acad Sci 1999;874:222-35.

\section{STAMPS IN CARDIOLOGY}

\section{History of cardiac surgery}

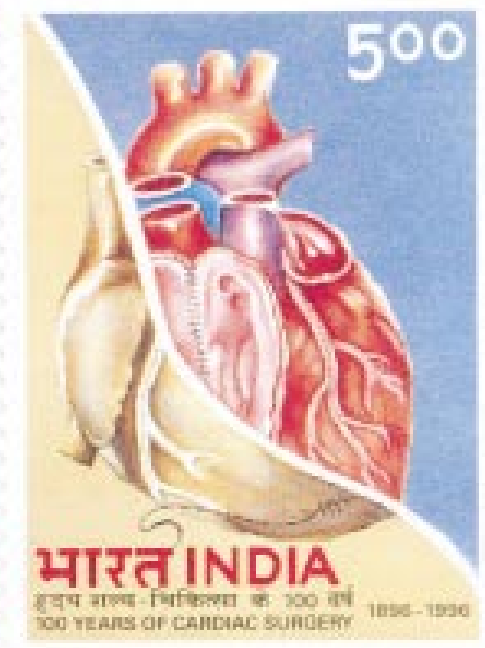

O 25 February 1996 India released this stamp to commemorate 100 years of cardiac surgery. The brochure issued by the Department of Posts contains the following comments: Stephen Paget, a famous British surgeon, remarked in his textbook on surgery of the chest as late as in 1896, 'Surgery of the heart has probably reached the limit set by nature to all surgery; no new method and no new discovery can overcome the natural difficulties that attend a wound of the heart'. The same year however, Ludwig Rehn of Frankfurt performed the first successful repair of a stab wound of the heart. The era of cardiac surgery had begun". The following cardiac surgical milestones over the next 100 years are quoted: surgery on the aortic valve (Theodore Tuffier, Paris 1912), surgery on the mitral valve (Elliot Cutter, Boston 1923), systemic pulmonary shunt for blue babies (Alfred Blalock, Baltimore 1945), closure of atrial septal defect (John Lewis, Minnesota 1952), valve substitute implantation (Charles Hufnagel, Georgetown 1952), open heart operation (John Gibbon, Boston 1953), totally implantable pacemaker (Ake Senning, Sweden 1959), successful coronary artery bypass (Michael DeBakey, Houston 1964), and cardiac transplantation (Christiaan Barnard, Cape Town 1967).

The design of the stamp has been very carefully chosen to mark 100 years of cardiac surgery. On the left part of the design is depicted the first ever successful suturing of a heart wound and on the right cardiac transplantation in progress. A total of 600000 stamps were printed by Calcutta Security Printers using the process of photo offset. 